\title{
Microsurgical decompression of tumor of a rare posterior third ventricular pathology
}

\author{
Anil Nanda, MD, MPH, Subhas Konar, MD, Piyush Kalakoti, MD, and Tanmoy Maiti, MD \\ Neurosurgery, Louisiana State University Health Sciences Center, Shreveport, Louisiana \\ Of the posterior third ventricular tumors, a papillary tumor of the pineal gland is a rare entity that originates from special- \\ ized ependymoma of the subcommissural organ. In this video narration, we present a case of a 33-year-old male with \\ headaches and recent cognitive decline due to a posterior third ventricular lesion. The patient underwent a posterior \\ interhemispheric approach, and a gross-total decompression was achieved with no signs of recurrence in a 2-year \\ follow-up period. With this case we highlight the microsurgical technique employed for decompressing tumors of the \\ posterior third ventricular region with preservation of eloquent structures and draining veins.
}

The video can be found here:https://youtu.be/o0WbyOqmtX0.

KEYWORDS microsurgical decompression; occipital interhemispheric; pineal mass; papillary tumor; posterior third ventricular pathology; video 